\title{
Polifarmácia em idosos do programa universidade aberta à terceira idade
}

\section{Polypharmacy in elderly at program university opened for seniors}

\author{
Niedja Maria Coelho Alves $^{1}(\mathbb{D})$, Albanita Gomes da Costa de Ceballos ${ }^{2}$ (D) \\ 1. Programa de Pós-graduação Integrado em Saúde Coletiva da Universidade Federal de Pernambuco (UFPE), Recife, PE, Brasil. 2. Docente do Departamento \\ de Medicina Social da Universidade Federal de Pernambuco (UFPE), Recife, PE, Brasil.
}

\section{Resumo}

Introdução: Polifarmácia é o uso de dois ou mais medicamentos para um ou vários problemas de saúde. Sua prática pode acarretar reações adversas e interações medicamentosas, principalmente em idosos, que são os mais vulneráveis a esses problemas. Objetivo: descrever a polifarmácia em idosos inscritos no Programa Universidade Aberta à Terceira Idade (UnATI) da Universidade Federal de Pernambuco (UFPE). Métodos: estudo do tipo transversal descritivo, realizado com alunos matriculados na UnATI/UFPE, os quais responderam a um questionário sobre utilização de medicamentos e características sociodemográficas. Resultados: dos 159 pesquisados, a maioria dos idosos estava entre 60 a 70 anos de idade, com renda média familiar de 1,4 a 4,4 salários mínimos, segundo grau completo, tendo realizado até três consultas médicas nos últimos 12 meses, possuía doenças crônicas, sendo hipertensão arterial, diabetes e colesterol alto as mais citadas. Quanto ao uso de medicamentos, a maioria não praticou automedicação, e a polifarmácia ocorreu em 78\%, sendo a polifarmácia menor mais prevalente que a maior. Conclusão: devido à grande frequência de uso de medicamentos e suas potenciais consequências para a saúde, é preciso investir na educação em saúde visando ao uso racional de medicamentos pelos idosos.

Palavras-chave: Idoso. Uso de medicamentos. Polifarmácia.

\begin{abstract}
Introduction: Polypharmacy is the use of two or more drugs for one or multiple health problems. This practice can cause to adverse drug reactions and interactions, especially in the elderly who are most vulnerable to these problems. Objective: to describe polypharmacy in the elderly registered in the Open University for the Third Age (UnATI) of the Federal University of Pernambuco (UFPE). Methods: a descriptive cross-sectional study, conducted with students in UnATI/UFPE, which answered a questionnaire about drug use and sociodemographic characteristics. Results: among 159 respondents, the majority of the individuals were 60-70 years old, average family income from 1.4 to 4.4 minimum wages, high school graduates, held up three physician visits in the last 12 months, chronic diseases, hypertension, diabetes and high cholesterol were the most cited. Regarding the use of drugs, most of them did not practice self-medication and polypharmacy occurred in $78 \%$, the smallest polypharmacy more prevalent than most. Conclusion: due to the high frequency of drug use, we need to invest in health education to encourage rational use of drugs by the elderly.
\end{abstract}

Key words: Aged. Drug utilization. Polypharmacy.

\section{INTRODUÇÃO}

O crescimento da população idosa é um fenômeno mundial e, no Brasil, as modificações do perfil demográfico têm ocorrido de forma acelerada. Os resultados da Pesquisa Nacional por Amostra de Domicílios (PNAD), de 2016, mostram que o número de pessoas com 60 ou mais anos de idade passou de 25,5 milhões para 29,6 milhões entre os anos de 2012 e $2016^{1}$. Essa transição demográfica representa uma conquista significativa em termos de qualidade de vida, mas, ao mesmo tempo, um grande desafio para o sistema de saúde do país.

O processo de envelhecimento é acompanhado de uma maior demanda pelos serviços de saúde e por medicamentos, o que predispõe a população geriátrica aos efeitos adversos de medicamentos, seja pela prática da polifarmácia ou polimedicação (uso simultâneo de vários medicamentos) ou da automedicação, a qual pode ser agravada nos praticantes da polifarmácia².
As peculiaridades do envelhecimento, como o aumento das enfermidades crônico degenerativas, favorecem a prescrição e o uso de múltiplos medicamentos na população idosa ${ }^{3}$, ao mesmo tempo em que as alterações farmacocinéticas e farmacodinâmicas inerentes ao envelhecimento ${ }^{4}$ influenciam na vulnerabilidade dos idosos aos problemas decorrentes do uso de medicamentos ${ }^{2}$.

A prática da polifarmácia é um dos tipos mais comuns de uso irracional de medicamentos e está associada ao aumento do risco e da gravidade das reações adversas a medicamentos, como precipitar interações medicamentosas, causar toxicidade cumulativa, ocasionar erros de medicação, reduzir a adesão ao tratamento e elevar a morbimortalidade. Assim, essa prática se relaciona diretamente aos custos assistenciais, que incluem medicamentos, e as repercussões advindas desse uso ${ }^{4}$ O Sistema Nacional de Informações Tóxico-Farmacológicas

Correspondência: Albanita Ceballos - Universidade Federal de Pernambuco, Centro de Ciências da Saúde - Departamento de Medicina Social. Av. da Engenharia, s/n, Bloco D - 1ํo andar. Cidade Universitária - Recife, Pernambuco. CEP 50.740-600. albanitagcceballos@gmail.com

Conflito de interesse: Não há conflito de interesse por parte de qualquer um dos autores.

Recebido em: 6 Dez 2017; Revisado em: 11 Jun 2018; 6 Ago 2018; Aceito em: 9 Ago 2018 
(SINITOX) registrou, em 2015, a ocorrência de 2.070 (8,42\%) casos de intoxicação por medicamentos entre idosos de todos os Estados do Brasil ${ }^{5}$.

Embora não seja um termo consensual, é possível definir polifarmácia como o uso de múltiplas doses ou medicamentos para um ou vários problemas de saúde ${ }^{6}$. Pode ser desenvolvida a partir da contribuição de fatores como o fácil acesso aos medicamentos, a baixa frequência de uso de recursos não farmacológicos para o manejo de problemas médicos, as características de pacientes e médicos bem como a percepção destes sobre o conceito de saúde?.

A população idosa chega a constituir $50 \%$ dos multiusuários de medicamentos que, em sua maioria, são prescritos para problemas como doenças cardiovasculares, respiratórias, neoplasias, diabetes mellitus, distúrbios do trato gastrintestinal, perturbações psicológicas, entre outras ${ }^{6}$. Estudos mostram prevalências de uso de medicamentos e de polifarmácia na população idosa de $83 \%$ e $35,4 \%$, respectivamente ${ }^{8}$.

Considerando que a população idosa no Brasil convive com problemas de longa duração, utiliza frequentemente os serviços de saúde e se constitui como maior consumidora de medicamentos, o objetivo deste estudo é descrever a polifarmácia em idosos, de acordo com características sociodemográficas e relacionadas à saúde geral.

\section{MÉTODOS}

Em 2012, a população do Estado de Pernambuco foi estimada em 8.981.212 habitantes, sendo $1.090 .627 \mathrm{com} 60$ ou mais anos de idade, dos quais, 192.943 idosos estavam em Recife.

Para atender à população idosa, foi criada, em 1996, a Universidade Aberta à Terceira Idade na Universidade Federal de Pernambuco (UnATI-UFPE), a qual representa um espaço de convivência em grupo com objetivo de promoção e incentivo de ações para a melhoria de sua qualidade de vida?

O presente estudo, do tipo transversal observacional, foi desenvolvido com 159 idosos não institucionalizados e capazes de se comunicar, matriculados na UnATI-UFPE, entre os meses de setembro e dezembro de 2013, convidados durante os intervalos de cursos de yoga, italiano, espanhol, informática, pintura e ética e selecionados por sorteio.

O cálculo amostral foi realizado no módulo StatCalc do Epiinfo ${ }^{\circledR}$, versão 7.0 para o estudo de corte seccional e os parâmetros de poder do estudo $80 \%$, Intervalo de Confiança de $95 \%$ e frequência do desfecho $51,9 \%^{6}$. A amostra final calculada foi igual a 159 sujeitos.

O questionário foi elaborado pela equipe da pesquisa, com três blocos de questões: perfil sociodemográfico, saúde geral e medicação utilizada. Os dados foram processados no programa Epiinfo ${ }^{\circledR}$, versão 7.0 em que foram estudadas as frequências das variáveis e feita a análise bivariada das variáveis segundo a polifarmácia. Para analisar a polifarmácia, foram criadas três variáveis: Polifarmácia Geral (uso de dois ou mais medicamentos); Menor (2 a 4 medicamentos) e Maior (5 ou mais).

Após anuência da UNATI/UFPE, o projeto foi submetido ao Comitê de Ética em Pesquisa do Centro de Ciências da Saúde da UFPE e aprovado sob o Parecer de número 853.920. Foi assegurada a confidencialidade dos dados fornecidos para a presente pesquisa. Os entrevistados assinaram o TCLE, seguindo as recomendações da Resolução 466/2012.

\section{RESULTADOS}

Verificou-se que a maioria dos pesquisados tinha entre 60 e 70 anos de idade, era do sexo feminino, sem companheiro, e que morava com pessoas da família, com renda entre $\mathrm{R} \$ 1.001,00$ e $\mathrm{R} \$ 3.000,00$ e com o segundo grau/ensino médio completo (Tabela 1).

Tabela 1 Descrição da população segundo variáveis sociodemográficas, Recife, PE,2014

\begin{tabular}{lrr}
\hline Variável & N (159) & $\%$ \\
\hline Idade & & \\
60 a 70 anos & 100 & 62,9 \\
71 ou mais anos & 59 & 37,1
\end{tabular}

Sexo

$\begin{array}{lrr}\text { Masculino } & 7 & 4,4 \\ \text { Feminino } & 152 & 95,6\end{array}$

Situação conjugal

Com companheiro $\quad 45 \quad 28,3$

Sem companheiro $\quad 114 \quad 71,7$

Com quem mora

Com família $\quad 98 \quad 61,6$

Com outros $16 \quad 10,1$

Sozinho $45 \quad 28,3$

Renda média declarada do domicílio

600,00 a $1.000,00 \quad 37 \quad 25,3$

$1.001,00$ a $3.000,00 \quad 70 \quad 47,9$

$3.001,00$ a $5.000,00 \quad 2517,1$

$5.001,00$ a $20.000,00 \quad 14 \quad 9,6$

Número de pessoas dependentes da renda

Uma ou duas 10364,8

\begin{tabular}{lll} 
Mais de duas & $56 \quad 35,2$ \\
\hline
\end{tabular} 


\begin{tabular}{lrc}
\hline Variável & N (159) & $\%$ \\
\hline Escolaridade & & \\
Até 1ํo grau completo & 42 & 26,4 \\
Até 2o grau completo & 63 & 39,6 \\
Até Superior completo & 54 & 34,0 \\
Raça/Cor & & \\
Preta ou parda & 84 & 52,8 \\
Branca ou outras & 75 & 47,2 \\
\hline
\end{tabular}

Quanto à saúde geral da população estudada, os idosos referiram ter feito de uma a três consultas médicas nos últimos 12 meses. A maioria referiu possuir convênio ou plano de saúde e adquirir seus medicamentos apenas em farmácias privadas. As doenças crônicas mais referidas foram a hipertensão arterial $(70,6 \%)$, o diabetes mellitus, a hipercolesterolemia e a artrose (Tabela 2).

Tabela 2. Descrição da população segundo situação geral de saúde, Recife, PE, 2014

\begin{tabular}{|c|c|c|}
\hline Variável & N (159) & $\%$ \\
\hline \multicolumn{3}{|c|}{$\begin{array}{l}\text { Número de consultas médicas nos últimos } 12 \\
\text { meses (somente ambulatório) }\end{array}$} \\
\hline Nenhuma & 6 & 3,8 \\
\hline Uma a três & 92 & 57,9 \\
\hline Quatro ou mais & 61 & 38,4 \\
\hline \multicolumn{3}{|l|}{$\begin{array}{l}\text { Número de vezes que procurou serviços } \\
\text { de saúde nos últimos } 12 \text { meses (Ex: } \\
\text { emergências) }\end{array}$} \\
\hline Não procurou & 71 & 44,7 \\
\hline Uma a três vezes & 68 & 42,8 \\
\hline Quatro ou mais vezes & 20 & 12,6 \\
\hline \multicolumn{3}{|l|}{ Serviços de saúde que utiliza } \\
\hline SUS & 47 & 29,6 \\
\hline Convênio/Plano de saúde & 87 & 54,7 \\
\hline SUS e Convênio/Plano & 25 & 15,7 \\
\hline \multicolumn{3}{|l|}{ Onde adquire medicamentos } \\
\hline Farmácias exclusivas do SUS & 17 & 10,3 \\
\hline Farmácias do SUS e privadas & 64 & 40,3 \\
\hline Farmácias exclusivas da rede privada & 78 & 49,1 \\
\hline \multicolumn{3}{|l|}{ Uso de bebida alcoólica } \\
\hline $\operatorname{Sim}$ & 22 & 13,8 \\
\hline Não & 137 & 86,2 \\
\hline \multicolumn{3}{|l|}{ Fumante } \\
\hline Sim & 3 & 1,9 \\
\hline Não & 156 & 98,1 \\
\hline \multicolumn{3}{|c|}{$\begin{array}{l}\text { Frequência da prática de atividades físicas na } \\
\text { semana }\end{array}$} \\
\hline Quatro a sete vezes & 53 & 33,3 \\
\hline
\end{tabular}

J. Health Biol Sci. 2018; 6(4): 412-418

\begin{tabular}{lrr}
\hline Variável & N (159) & \% \\
\hline Uma a três vezes & 64 & 40,3 \\
Não pratica & 42 & 26,4 \\
Doença Crônica & & \\
Sim & 136 & 85,5 \\
Não & 23 & 14,5 \\
Doenças crônicas mais prevalentes & & \\
Hipertensão arterial & 96 & 70,6 \\
Diabetes & 38 & 27,9 \\
Hipercolesterolemia & 35 & 25,7 \\
Artrose & 17 & 12,5 \\
\hline
\end{tabular}

Quanto ao uso de medicamentos, a maior parte da amostra referiu não se automedicar, mas utilizar dois ou mais medicamentos para um ou vários problemas de saúde $(78,0 \%)$, sendo que, destes, $62,3 \%$ utilizaram de dois a quatro produtos e $15,7 \%$, cinco ou mais. Os principais medicamentos para doenças crônicas foram a Sinvastatina, a Losartana potássica, os ansiolíticos da classe dos benzodiazepínicos, os antidepressivos e o Atenolol (Tabela 3).

Tabela 3. Descrição da população segundo perfil de utilização de medicamentos, Recife, PE,2014

\begin{tabular}{lrr}
\hline Variável & N (159) & \% \\
\hline Automedicação & 25 & 15,7 \\
Sim & 134 & 84,3 \\
Não & & \\
Polimedicação Geral & 124 & 78,0 \\
Sim & 35 & 22,0 \\
Não & & \\
Polimedicação menor & 99 & 62,3 \\
Sim & 60 & 37,7 \\
Não & & \\
Polimedicação maior & 25 & 15,7 \\
Sim & 134 & 84,3 \\
Não & & \\
Principais medicamentos de uso crônico & 28 & 17,6 \\
Sinvastatina & 27 & 16,9 \\
Losartana Potássica & 9 & 5,7 \\
Atenolol & 15 & 9,4 \\
\hline Ansiolíticos (BDZ) & & \\
\hline
\end{tabular}

O estudo mostrou que os que praticam polimedicação estão na faixa de idade entre 60 a 70 anos, com companheiro, com renda domiciliar declarada que varia de 1,4 a 4,4 salários mínimos, com ensino médio concluído; consultaram o médico quatro ou mais vezes nos últimos 12 meses, utilizaram convênio ou planos de saúde e adquiriram seus medicamentos tanto em farmácias públicas quanto em privadas (Tabelas 4 e 5 ). 
Tabela 4. Descrição da polifarmácia segundo variáveis sociodemográficas, Recife, PE,2014

\begin{tabular}{|c|c|c|c|c|c|c|c|c|}
\hline \multirow{3}{*}{ Variável } & \multicolumn{8}{|c|}{ Polimedicação } \\
\hline & \multicolumn{2}{|c|}{ Sem polimedicação } & \multicolumn{2}{|c|}{ Geral } & \multicolumn{2}{|c|}{ Menor } & \multicolumn{2}{|c|}{ Maior } \\
\hline & $\mathbf{N}$ & $\%$ & $\mathbf{N}$ & $\%$ & $\mathbf{N}$ & $\%$ & $\mathbf{N}$ & $\%$ \\
\hline \multicolumn{9}{|l|}{ Idade } \\
\hline 60 a 70 & 20 & 20,0 & 80 & 80,0 & 63 & 79,0 & 17 & 21,0 \\
\hline 71 ou mais & 15 & 25,4 & 44 & 74,6 & 36 & 81,8 & 8 & 18,2 \\
\hline \multicolumn{9}{|l|}{ Sexo } \\
\hline Masculino & 2 & 28,6 & 5 & 71,4 & 3 & 60,0 & 2 & 40,0 \\
\hline Feminino & 33 & 21,7 & 119 & 78,3 & 96 & 80,7 & 23 & 1,3 \\
\hline \multicolumn{9}{|l|}{ Situação Conjugal } \\
\hline Com companheiro & 9 & 20,0 & 36 & 80,0 & 27 & 75,0 & 9 & 25,0 \\
\hline Sem companheiro & 26 & 22,8 & 88 & 77,2 & 72 & 81,8 & 16 & 18,2 \\
\hline \multicolumn{9}{|l|}{ Com quem mora } \\
\hline Com família & 21 & 21,4 & 77 & 78,6 & 58 & 75,3 & 19 & 24,7 \\
\hline Com outros & 4 & 25,0 & 12 & 75,0 & 10 & 83,3 & 2 & 4 \\
\hline Sozinho & 10 & 22,2 & 35 & 77,8 & 31 & 88,6 & 4 & 11,4 \\
\hline \multicolumn{9}{|c|}{ Renda média declarada do domicílio (R\$) } \\
\hline $600-1.000$ & 11 & 29,7 & 26 & 70,3 & 19 & 73,0 & 7 & 27,0 \\
\hline $1.001-3000$ & 13 & 18,6 & 57 & 81,4 & 45 & 79,0 & 12 & 21,0 \\
\hline $3001-5000$ & 6 & 24,0 & 19 & 76,0 & 17 & 89,5 & 2 & 10,5 \\
\hline $5001-20000$ & 3 & 21,4 & 11 & 78,6 & 9 & 81,8 & 2 & 18,2 \\
\hline \multicolumn{9}{|c|}{ № de dependentes da renda } \\
\hline Uma ou duas & 22 & 21,4 & 81 & 78,6 & 66 & 81,5 & 15 & 18,5 \\
\hline Mais de duas & 13 & 23,2 & 43 & 76,8 & 33 & 76,7 & 10 & 23,3 \\
\hline \multicolumn{9}{|l|}{ Escolaridade } \\
\hline Até 1 ㅇg grau completo & 12 & 28,6 & 30 & 71,4 & 23 & 76,7 & 7 & 23,3 \\
\hline Até 20 grau completo & 12 & 19,0 & 51 & 81,0 & 41 & 80,4 & 10 & 19,6 \\
\hline Até Superior completo & 11 & 20,4 & 43 & 79,6 & 35 & 81,4 & 8 & 18,6 \\
\hline \multicolumn{9}{|l|}{ Raça/Cor } \\
\hline Preta ou parda & 18 & 21,4 & 66 & 78,6 & 51 & 77,3 & 15 & 22,7 \\
\hline Branca ou outras & 17 & 22,7 & 58 & 77,3 & 48 & 82,8 & 10 & 17,2 \\
\hline
\end{tabular}


Tabela 5. Descrição da polifarmácia segundo a situação geral de saúde, Recife, PE,2014

\begin{tabular}{|c|c|c|c|c|c|c|c|c|}
\hline \multirow{3}{*}{ Variável } & \multicolumn{8}{|c|}{ Polimedicação } \\
\hline & \multicolumn{2}{|c|}{$\begin{array}{r}\text { Sem } \\
\text { polimedicação }\end{array}$} & \multicolumn{2}{|c|}{ Geral } & \multicolumn{2}{|c|}{ Menor } & \multicolumn{2}{|c|}{ Maior } \\
\hline & $\mathbf{N}$ & $\%$ & $\mathbf{N}$ & $\%$ & $\mathbf{N}$ & $\%$ & $\mathbf{N}$ & $\%$ \\
\hline \multicolumn{9}{|c|}{$\begin{array}{l}\text { Número de consultas médicas nos últimos } 12 \text { meses } \\
\text { (somente ambulatório) }\end{array}$} \\
\hline Nenhuma & 4 & 66,7 & 2 & 33,3 & 2 & 100,0 & 0 & - \\
\hline Uma a três & 24 & 26,1 & 68 & 73,9 & 56 & 82,4 & 12 & 17,6 \\
\hline Quatro ou mais & 7 & 11,5 & 54 & 88,5 & 41 & 76,0 & 13 & 24,0 \\
\hline \multicolumn{9}{|c|}{$\begin{array}{l}\text { Número de vezes que procurou serviços de saúde nos } \\
\text { últimos } 12 \text { meses (Ex.: emergências) }\end{array}$} \\
\hline Não procurou & 19 & 26,8 & 52 & 73,2 & 42 & 80,8 & 10 & 19,2 \\
\hline Uma a três vezes & 14 & 20,6 & 54 & 79,4 & 46 & 85,2 & 8 & 14,8 \\
\hline Quatro ou mais vezes & 2 & 10,0 & 18 & 90,0 & 11 & 61,1 & 7 & 38,9 \\
\hline \multicolumn{9}{|l|}{ Serviços de saúde que utiliza } \\
\hline SUS & 12 & 25,5 & 35 & 74,5 & 25 & 71,4 & 10 & 28,6 \\
\hline Convênio & 4 & 16,1 & 73 & 83,9 & 61 & 83,6 & 12 & 16,4 \\
\hline SUS e convênio & 9 & 36,0 & 16 & 64,0 & 13 & 81,2 & 3 & 18,8 \\
\hline \multicolumn{9}{|l|}{ Onde adquire medicamentos } \\
\hline Farmácias exclusivas do SUS & 7 & 41,2 & 10 & 58,8 & 7 & 70,0 & 3 & 30,0 \\
\hline Farmácias do SUS e privadas & 8 & 12,5 & 56 & 87,5 & 41 & 73,2 & 15 & 26,8 \\
\hline Farmácias exclusivas da rede privada & 20 & 25,6 & 58 & 74,4 & 51 & 88,0 & 7 & 12,0 \\
\hline \multicolumn{9}{|l|}{ Uso de bebida alcoólica } \\
\hline Sim & 7 & 31,8 & 15 & 68,2 & 12 & 80,0 & 3 & 20,0 \\
\hline Não & 28 & 20,4 & 109 & 79,6 & 87 & 79,8 & 22 & 20,2 \\
\hline \multicolumn{9}{|l|}{ Fumante } \\
\hline Sim & 2 & 66,7 & 1 & 33,3 & 0 & & 1 & 100,0 \\
\hline Não & 33 & 21,2 & 123 & 78,8 & 99 & 80,5 & 24 & 19,5 \\
\hline \multicolumn{9}{|c|}{ Frequência da prática de atividades físicas na semana } \\
\hline Quatro a sete vezes & 12 & 22,6 & 41 & 77,4 & 33 & 80,5 & 8 & 19,5 \\
\hline Uma a três vezes & 16 & 25,0 & 48 & 75,0 & 38 & 79,2 & 10 & 20,8 \\
\hline Não pratica & 7 & 16,7 & 35 & 83,3 & 28 & 80,0 & 7 & 20,0 \\
\hline \multicolumn{9}{|l|}{ Doenças crônicas } \\
\hline Não & 17 & 73,9 & 6 & 26,1 & 6 & 100,0 & 0 & - \\
\hline Sim & 18 & 13,2 & 118 & 86,8 & 93 & 78,8 & 25 & 21,2 \\
\hline
\end{tabular}

\section{DISCUSSÃO}

A análise dos dados sociodemográficos demonstrou que a maioria dos idosos participantes do Programa UnATI/UFPE era do sexo feminino, com faixa etária entre 60 e 70 anos e sem companheiro, resultados também verificados em estudo realizado em áreas urbanas e rurais no Sul do Brasil ${ }^{10}$. A expectativa de vida das mulheres brasileiras em relação aos homens é de 7,3 anos maior ${ }^{11}$, o que explica a quantidade maior de viúvas, que, por sua vez, são as principais participantes de grupos e atividades voltadas ao segmento idoso.

Sobre a renda domiciliar declarada, houve variação de 1,4 a 4,4 salários mínimos, enquanto que, em estudo realizado em 2003, também no contexto da UnATI/UFPE, a renda estava entre 7 e 10 salários $^{12}$. É possível que, nesta pesquisa, os idosos 
tenham omitido informações sobre outros rendimentos além da aposentadoria, talvez por falta de clareza na pergunta ou por uma mudança real no perfil do público da UnATI/UFPE. É importante frisar que, em ambos os estudos, a maioria concluiu o segundo grau, mas houve um aumento da quantidade de idosos com nível superior no presente estudo.

Um inquérito domiciliar realizado em Recife-PE, no ano de 2009 , verificou que $64,5 \%$ dos idosos pesquisados utilizavam, exclusivamente, a Estratégia Saúde da Família (ESF)13, resultado discordante do observado neste estudo, em que a procura exclusiva pelo Sistema Único de Saúde ocorreu em 29,6\%. É possível pensar que, apesar da queda de renda declarada dos participantes da UnATI/UFPE, a compra desses seguros, ou a utilização de convênios de familiares como dependentes, signifique a garantia de melhores formas de acesso à assistência, além de maior heterogeneidade sociodemográfica verificada nesta amostra, composta de pessoas preocupadas com o bemestar.

A existência de, pelo menos, uma doença crônica foi observada na maioria da amostra, corroborando a prevalência observada anteriormente em Recife-PE ${ }^{13}$. Para Miranda et al $(2016)^{14}$, o cenário atual de envelhecimento demonstra a necessidade das ações de promoção de saúde e prevenção de doenças, visando evitar ou retardar as doenças crônicas e as incapacidades.

A não prática de atividades físicas foi encontrada em 58,5\% de entrevistados em Tatuí-SP ${ }^{15}$, enquanto que, neste estudo, declararam-se não adeptos dessa prática apenas 26,4\%. Esse resultado pode ser explicado pelo incentivo dado pela própria UnATI para a prática de atividade física por meio do oferecimento de cursos de atividades físicas ou esportivas como Yoga e Pilates.

O fenômeno da automedicação foi vivenciado por 15,7\% dos alunos nesta pesquisa, resultado superior aos 6,7\% encontrados em Recife, em $2013^{13}$, e inferior aos 44,2\% verificados em estudo com idosos do interior baiano ${ }^{16}$. A discrepância desses resultados pode ser explicada pela diferente significação da automedicação. Ou seja, alguns grupos consideram como automedicação o uso de chás, unguentos, lambedores e beberagens; outros grupos consideram o uso de medicamentos que não precisam de prescrição, como analgésicos e antigripais, e ainda outros consideram como automedicação apenas quando fazem uso de medicamentos que necessitam de prescrição médica. É preciso padronizar a forma de aferição da variável para criar seus critérios de comparabilidade em diferentes cenários.

Os idosos que mais se polimedicaram foram os da faixa etária de 60 a 70 anos e os sem companheiro, resultados também observados no estudo em Recife-PE, no ano de $2013^{13}$. A polimedicação maior, considerada neste trabalho com o uso concomitante de cinco ou mais medicamentos, foi relatada por $15,7 \%$ dos alunos entrevistados, resultado próximo aos $11 \%$ e $13,9 \%$ descritos respectivamente em Recife-PE ${ }^{13}$ e na Região Sul do Brasil $^{10}$. No estudo de Cuentro et al $(2016)^{17}$, a média de medicamentos utilizados foi de 6,4 por idoso.

Entre os medicamentos de uso crônico mais utilizados, destacam-se os ansiolíticos da classe dos benzodiazepínicos, com 9,4\%, semelhante ao resultado de 9,3\% obtido em estudo sobre a utilização de drogas psicoativas por idosos de comunidade brasileira ${ }^{18}$. Apesar desse uso verificado, a ansiedade não consta entre as doenças mais prevalentes, o que poderia ser explicado pela dificuldade em assumir ou referir problemas mentais ou psicológicos existentes ou, por outro lado, pela prescrição desnecessária dessa classe que configura entre os medicamentos potencialmente inapropriados aos idosos ${ }^{19}$.

Apesar das limitações próprias de um estudo transversal e descritivo e de não ser possível, a partir dessa amostra, fazer inferências sobre o padrão de uso de medicamentos para idosos de outros grupos populacionais, acredita-se que os dados obtidos possam contribuir para a caracterização da utilização de medicamentos pelos idosos matriculados na UnATI/UFPE.

\section{CONCLUSÃO}

A polifarmácia está presente na vida dos idosos pesquisados, sendo a polifarmácia menor mais observada do que a maior. O uso de dois a quatro medicamentos é condizente com a prevalência de doenças crônicas, que, por sua vez, tornam os idosos mais vulneráveis aos possíveis efeitos indesejados causados pelo uso concomitante de medicamentos. Diante disso, acredita-se que é necessário investir em espaços de educação em saúde para promover o uso racional de medicamentos, bem como a otimização da farmacoterapia dispensada aos idosos.

\section{REFERÊNCIAS}

1. PNAD 2016: população idosa cresce 16,0\% frente a 2012 e chega a 29,6 milhões [internet]. Rio de janeiro; 2017 [acesso 2018 Jun 13]. Disponível em: https://agenciadenoticias.ibge.gov.br/agencia-noticias/2013-agencia-denoticias/releases/18263-pnad-2016-populacao-idosa-cresce-16-0-frente-a2012-e-chega-a-29-6-milhoes.html

2. Sá $M B$, Barros JAC, Sá MPBO. Automedicação em idosos na cidade de Salgueiro-PE. Rev. bras. epidemiol. 2007 Mar; 10(1):75-85. doi: http://dx.doi. org/10.1590/S1415-790X2007000100009.

3. Lyra DP Jr, Neves AS, Cerqueira KS, Marcellini PS, Marques TC, Barros JAC.
Influência da propaganda na utilização de medicamentos em um grupo de idosos atendidos em uma unidade básica de saúde em Aracaju (SE, Brasil). Ciênc. saúde coletiva. 2010 Nov; 15 (Supl 3): 3497- 3505.

4. SECOLI, SR. Polifarmácia: interações e reações adversas no uso de medicamentos por idosos. Rev. bras. enferm. 2010 Jan-Feb; 63(1): 136-40. doi: http://dx.doi.org/10.1590/S0034-71672010000100023.

5. Sistema Nacional de Informações Tóxico-Farmacológicas (SINITOX). Casos de Intoxicação por medicamentos por Unidade Federada, segundo faixa etária registrado em 2015 [internet]. Rio de Janeiro; 2015 [acesso 2018 Jun 13]. 
Disponível em: https://sinitox.icict.fiocruz.br/dados-nacionais.

6. Galato D, Silva ES, Tiburcio LS. Estudo de utilização de medicamentos em idosos residentes em uma cidade do sul de Santa Catarina (Brasil): um olhar sobre a polimedicação. Cienc. saúde coletiva. 2010 Sept;15(6): 2899-905. doi: http://dx.doi.org/10.1590/S1413-81232010000600027.

7. Almeida OP, Ratto L, Garrido R, Tamai S. Fatores preditores e consequências clínicas do uso de múltiplas medicações entre idosos atendidos em um serviço ambulatorial de saúde mental. Rev Bras. Psiquiatr. 1999 Set; 21(3): 152-7. doi: http://dx.doi.org/10.1590/S1516-44461999000300006.

8. Silva AL, Ribeiro AQ, Klein $\mathrm{CH}$, Acurcio FA. Utilização de medicamentos por idosos brasileiros, de acordo com a faixa etária: um inquérito postal. Cad. Saúde Pública. 2012 Jun; 28(6): 1033-45. doi: http://dx.doi.org/10.1590/S0102311X2012000600003.

9. Folder Universidade Aberta à Terceira Idade (UnATI). Universidade Federal de Pernambuco. Pró- Reitoria de Extensão. É preciso maiores informações para areferência.

10. Da Pizzol TS, Pons ES, Hugo FN, Bozzetti MC, SousaMLR, Hilgert JB. Uso de medicamentos entre idosos residentes em áreas urbanas e rurais de município no Sul do Brasil: um estudo de base populacional. Cad. Saúde Pública. 2012 Jan; 28(1): 104-14. doi: http://dx.doi.org/10.1590/S0102-311X2012000100011.

11. Instituto Brasileiro de geografia e Estatística. Expectativa de vida em $2012 .$. Disponível em:http://teen.ibge.gov.br/noticias-teen Acesso em: 13/12/2014.

12. Barreto KML, Carvalho EMF, Falcão IV, Less FJD, Leit VMM. Perfil sócioepidemiológico demográfico das mulheres idosas da Unive rsidade Aberta à
Terceira Idade no estado de Pernambuco. Rev. Bras. Saúde Mater. Infant. 2003 JulSet; 3(3): 339-54. doi: http://dx.doi.org/10.1590/S1519-38292003000300013.

13. Neves SJF, Marques APO, Leal MCC, Diniz ASM, Medeiros TS, Arruda IKG. Epidemiologia do uso de medicamentos entre idosos em área urbana do Nordeste do Brasil. Rev. Saúde Pública. 2013;47(4): 759-67. doi: http://dx.doi. org/10.1590/S0034-8910.2013047003768.

14. Miranda GMD, Mendes ACG, Silva ALA. O envelhecimento populacional brasileiro: desafios e consequências sociais atuais e futuras. Rev. Bras. Geriatria e Gerontologia 2016;19(3): 507-519.

15. Cruz AV, Fulone I, Alcalá M, Fernandes AA, Montebelo MI, Lopes LC. Uso crônico de diazepam em idosos atendidos na rede pública em Tatuí-SP. Rev. ciênc. farm. Básica apl. 2006; 2(3): 259-67.

16. Sales AS, Sales MGS, Casotti CA. Perfil farmacoterapêutico e fatores associados à polifarmácia entre idosos de Aiquara, Bahia, em 2014. Epedemiol. Serv. Saúde. 2017 Jan-Mar; 26(1): 121-132.

17. Cuentro VS, Modesto T, Andrade MA, Silva MVS. Prevalência e fatores associados à polifarmácia entre idosos de um hospital público. Rev Contexto \& Saúde 2016 Jan-Jun; 16(30): 28-35.

18. Chaimowicz F, Ferreira TJXM, Miguel DFA. Use of psychoactive drugs and related falls among older people living in a community in Brazil. Rev. Saúde Pública. 2000 Dez; 34(6): 631-5. doi: http://dx.doi.org/10.1590/S003489102000000600011.

19. Gorzoni ML, Fabri RMA, Pires SL. Medicamentos potencialmente inapropriados para idosos. Rev Assoc Med Bras. 2012;58(4): 442-6.

\section{Como citar este artigo/How to cite this article:}

Alves NMC, Ceballos AGC. IPolifarmácia em idosos do programa universidade aberta à terceira idade. J Health Biol Sci. 2018 Jul-Set; 6(4):412-418. 